\title{
The Role of Typography in Differentiating Look-Alike/ Sound-Alike Drug Names
}

\author{
$\overline{\text { Sandra Gabriele }}$
}

\begin{abstract}
Until recently, when errors occurred in the course of caring for patients, blame was assigned to the healthcare professionals closest to the incident rather than examining the larger system and the actions that led up to the event. Now, the medical profession is embracing expertise and methodologies used in other fields to improve its own systems in relation to patient safety issues. This exploratory study, part of a Master's of Design thesis project, was a response to the problem of errors that occur due to confusion between look-alike/sound-alike drug names (medication names that have orthographic and/or phonetic similarities). The study attempts to provide a visual means to help differentiate problematic names using formal typographic and graphic cues. The FDA's Name Differentiation Project recommendations and other typographic alternatives were considered to address issues of attention and cognition. Eleven acute care nurses participated in testing that consisted of word-recognition tasks and questions intended to elicit opinions regarding the visual treatment of look-alike/sound-alike names in the context of a label prototype. Though limited in sample size, testing provided insight into the kinds of typographic differentiation that might be effective in a high-risk situation.
\end{abstract}

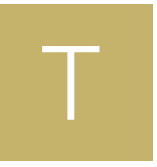

his paper reports on a portion of a Master's of Design in visual communication design thesis project. The larger study examines how information design (an area within visual communication design concerned with the clarity of information to facilitate understanding) might lessen medication error caused by confusion of look-alike/sound-alike drug names. The purpose of this exploratory study was to develop appropriate design proposals and testing protocols for evaluation and analysis. Included was the design and testing of medicine labels in terms of content (the presence or absence of information), composition/layout (positioning, prominence and grouping of information) and typography (choices regarding the visual attributes of typographic forms) for drugs that are repackaged in acute care hospital pharmacies and sent to nursing units. The portion of the study described here demonstrates how principles of typography might help to differentiate look-alike/sound-alike drug names.

\section{Designing for People: Human Factors and User- Centred Design}

For a number of years, human factors professionals have proven to be successful at looking into complex systems to deter- 
Figure 1. Contribution of packaging and labelling to medication errors

\begin{tabular}{|c|c|}
\hline Elements of Design & Packaging and Labelling Concerns \\
\hline Content & $\begin{array}{l}\text { - Inadequate warnings about proper drug use } \\
\text { - Prominence of company logos versus information that identifies the product }\end{array}$ \\
\hline $\begin{array}{l}\text { Composition/ } \\
\text { Layout }\end{array}$ & $\begin{array}{l}\text { - Lack of prominent placement of drug name and strength } \\
\text { - Insufficient prominence given to route of administration (e.g., nasal vs. injection, } \\
\text { intravenous vs. intramuscular) } \\
\text { - Poorly designed or cluttered labels } \\
\text { - Similar-appearing labels or packages of different products }\end{array}$ \\
\hline Typography & $\begin{array}{l}\text { - Small size and poor readability of printed information } \\
\text { - Lack of differentiation between drug products that have similar names }\end{array}$ \\
\hline Colour & - Poor use or absence of colour to differentiate products \\
\hline Visuals & Not Applicable \\
\hline
\end{tabular}

(Adapted from USP 1998)

mine root causes in aviation accidents and large-scale systems failures, such as nuclear disasters and blackouts. Healthcare providers, concerned with incidents of error, are taking measures to help reduce the occurrence of adverse events by making use of the practices of human factors engineering that have been successful in other domains (Kohn et al. 2000).

Information designers have concerns parallel to those of human factors specialists. Both are interested in how the end user interacts and behaves in relation to a designed artefact, whether it is a product, an environment or a visual communication. The information designer is concerned specifically with how his or her designs mediate communication for the user. Designers develop a "prediction" or potential solution with the intent to affect changes in a situation or user behaviour. In order to do this, the designer must become well acquainted with the problem and understand the user's information processing in relation to the product or system and the environment in which it is used (Popovic 1999).

Psychologist and author Donald Norman (1990) recognizes that human beings routinely make errors and that designers should bear this in mind. Norman believes designing with a user-centred philosophy allows for examination of the interaction between humans and their "machines." The success of this relationship is especially critical in any high-risk environment where errors can arise due to shortfalls on either side, contributing to accidents.

\section{Medication Errors: Why They Occur}

Professor James Reason (2000a), a leading authority on the topic of human error, believes that error in medicine can be viewed from a "person" approach or from a "system" approach. This is illustrated in a study conducted by the United States Food and Drug Administration (FDA 2002) in May 2001, in which reports of 265 cases of medication errors were reviewed and classified by cause. Human factors and communication accounted for $61 \%$ of incidents, while systems errors - labelling, packaging/design and name confusions - accounted for 39\% (Thomas et al. 2001). By understanding human cognitive and physical capabilities and limitations in relation to the visual communication materials involved during the medication process, there is an opportunity for designers to intervene with visual solutions that may help minimize the occurrence of error.

Labelling and packaging concerns were cited as contributing to medication errors in an analysis of the U.S. Pharmacopeia (USP) medication errors database in a one-year period beginning June 1, 1996 (USP 1998). To help identify areas where improvements might be made from a design perspective, items listed in this report were categorized into the elements that shape visual communication design materials: content, composition/layout, typography, colour and visuals (Figure 1). One of the factors that can contribute to the administration of an incorrect medication is the confusion that can arise with lookalike/sound-alike drug names, those where the name is close to 
another orthographically (in written form) or phonetically (in spoken form) (Figures 2, 3).

Figure 2. Orthographic similarity

\section{Hydroxyzine - Hydroxyzine \\ Hydralazine - Hydralazine}

Figure 3. Phonetic similarity

\section{Zantac-/Zæntæk/ \\ Zyrtec-|Zirtek/}

\section{Addressing the Problem of Look-Alike/Sound-Alike Drug Names}

In recognition of reported incidents of error involving 16 specific look-alike/sound-alike drug pairs, the FDA (2002) developed the Name Differentiation Project, a recommendation to pharmaceutical manufacturers to voluntarily change the appearance of these names. They suggest cueing a part of the look-alike/sound-alike names with a change from lowercase to uppercase characters or "tallman letters" (Figure 4). Based on a series of controlled laboratory experiments, Filik et al. (2006) recently reported, “... studies suggest that tall man letters can make similar names less confusable perceptually and can increase attention to high-risk drug names." The notion of visually differentiating names is a point of departure for this study, in which typographic variations were tested with acute care hospital nurses to see if cueing might help them to differentiate drug names.

Figure 4. Name Differentiation Project and use of "tallman letters"

\section{Bupropion - BuPROPion}

\section{Buspirone - BusPIRone}

Engaging and maintaining the attention of the nurse throughout the medication use process is crucial in ensuring the patient receives the correct drug. James Reason (2000b) states that slips and lapses occur while performing routine tasks and are a result of automatic, unconscious processes. These types of errors take place when attention is diverted, whether by a distraction in the immediate surroundings, by preoccupation with something, or because of some type of change in the current plan of action.

By understanding that attention span is selective, has a limited capacity based on the task at hand and does not usually last very long, designers can influence a user's behaviour. Because viewers are attracted by things that are distinctive and novel, visual strategies such as the use of contrast, making elements large, bold and clear and highlighting and isolating relevant information will help to attract attention and facilitate understanding (Pettersson 2001).

While the FDA's recommendation is a positive step toward avoiding name confusions and error in the differentiation of the original 16 name pairs, indiscriminate use of the basic principle by applying "tallman letters" to drugs that are not on the list might lead to further confusion if the cueing is inconsistent. In addition, typographic principles and legibility research suggest that varying the visual attributes of a typeface, other than changing the case, would more effectively help to differentiate names.

We recognize words through the interactive processing at the levels of feature, character and word, with input from a higher level of semantic information (McClelland and Rumelhart 1981; Larson 2004). This model supports the idea that attention to features might help show differentiation within look-alike/ sound-alike names and act as a comprehension cue. Observing some design characteristics of uppercase characters, one should note that their construction does not provide sufficient distinctiveness in features from character to character, because in form they are not as varied as their lowercase counterparts. Only seven characters in the uppercase set are made up of a combination of straight and curved strokes, as against fifteen in the lowercase set. Fifteen characters in the uppercase set are made up of straight lines, as opposed to eight in the lowercase set. This illustrates the lack of diversity in the design of uppercase characters - one reason why they are more difficult to discern. Some typefaces do not make a clear distinction between the lowercase "l" the uppercase "I" and the numeral "1," which might lead to further confusion with look-alike/sound-alike drug names (Figure 5). This problem is worsened by the resolution capability of the media on which it is printed or displayed. With low-end inkjet printers (a fairly standard type of printer used in hospital pharmacies) and computer monitors, the quality of small type can be extremely poor, making drug labels difficult to read with accuracy. Most typefaces designed for print don't automatically translate into legible text when used for screen applications. Only recently have typefaces designed specifically for this use become widely available. To compensate for technical short- 
comings, the typeface designer must consider the technology that will be used for print or electronic display.

Figure 5. Lack of distinction between lowercase "I," uppercase "I" and numeral " 1 "

Typeface - Bodoni Book

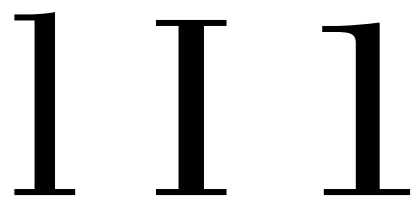

Typeface - Gill Sans

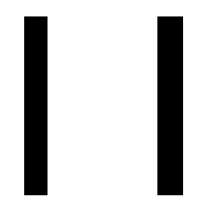

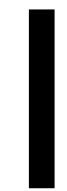

\section{Legibility and Contrast}

Perhaps a reason that a better alternative has not been considered is that, from a pharmacy perspective, limitations in computers, printers and existing software have not allowed for alternatives such as boldface characters or graphics. For many years, the only method of accentuating information was the use of all-uppercase characters (Figure 6). The continued usage is based on standard practices rather than what might work more effectively if considering principles that designers draw upon to accentuate information. While this study included the recommended change between lowercase and uppercase characters to differentiate names, as a point of departure and comparison, it looks further to find alternatives that may have more visual impact while maintaining legibility.

Design researcher Ellen Lupton (2003) notes that the field of typography "remains ruled, largely, by convention and intuition. ..." Studies on legibility are conducted for the most part by psychologists and human factors and human-computer interaction specialists. It is important to note that although legibility studies provide valuable information in particular contexts and under specific circumstances, they cannot be generalized with certainty and used indiscriminately in all situations. In line with a user-centred approach, final designs, containing the specific content, character sizes, line lengths, etc., require testing in their intended context and with the user in their work environment, performing a specific task. Even though they are limited in their applicability, studies in legibility, combined with the experience of the designer, can serve as a guide for the development of effective visual communications. Initial designs are often based on some combination of the designer's experience - what scientists might call a "hunch" - preliminary expert and user input and existing literature. Because design is an iterative process, interim assessments, testing and final evaluation are essential.

Figure 6. Use of uppercase characters for emphasis

\section{BUSPIRONE $5 \mathrm{mg}$ \\ $(1 / 2 \times 10 \mathrm{ng})$ \\ Generic for "BUSPAR" Control \#03 (pms) Exp: $05 / 02$}

Pharmacy-generated medication labels often use uppercase characters to show emphasis.

Contrast serves to punctuate, draw attention to and clarify elements by placing them in opposition to each other (Dair 1967; McCreight 1996). Typographic contrast is achieved by differences in the visual attributes displayed by type. These are, typestyle (serif or sans serif), typeface design or family, weight (light, medium, bold), stance (roman, italic), character width (regular, condensed, expanded) and case (uppercase, lowercase, small caps). Graphic devices (rules or lines, shapes) and spacing (character, word and line spacing) can be used in place of or in conjunction with contrasting type arrangements to highlight or cue text (Figure 7). When combined with verbal cues, meaningful or natural breaks that occur in text, they act as emphasis to capture attention and facilitate understanding. Typographic design strategies were guided by principles of typography and legibility research and by a visual exploration of variations that were assessed on their ability to create emphasis and contrast (Figure 8).

Final designs (Figure 9) selected for testing were based upon the desire to simplify the choice for participants while providing a variation in degree of contrast in terms of the "colour" of the text. In typographic terminology, colour refers to the grey value created in typeset text. Colour varies according to the visual attributes of a typeface and the amount of letter, word and line spacing (Ruegg 1989). For example, boldface text looks darker in colour than lightface. 
Figure 7. Visual attributes and graphic devices for distinguishing text

\begin{tabular}{ll} 
Visual Atributes & \\
\hline Typestyle & Serif \\
& Sans Serif \\
\hline Typeface & Garamond \\
& Bodoni \\
\hline weight & Light \\
& Medium \\
& Bold \\
\hline stance & Roman \\
& Italic \\
\hline Character & Condensed \\
width & Regular \\
& Expanded \\
\hline Case & UPPERCASE \\
& lowercase \\
& SMALL CAPS \\
\hline
\end{tabular}

The typographic variations consisted of

- The least extreme, a contrast in case - a change from lowercase characters to uppercase characters

- A middle ground, a contrast in weight - a change from medium-weight characters to boldface (lowercase) characters

- The most extreme, a contrast - a change from black characters to white characters on a solid black rectangle

\section{Testing Look-Alike/Sound-Alike Drug Names for Visual Attributes (Typography)}

This exploratory study made use of a mixed-method approach to testing, using both quantitative and qualitative data collection. Quantitative information was used to examine error rates for each of the variations, while qualitative information was used to compare attitudes and opinions. Participants were 11 acute care hospital nurses. Three word-recognition tests were conducted to compare how effective the contrasts might be in making names more memorable. Participants were given a stimulus list to examine for each test. Each contained seven look-alike/sound-alike names with one of the three typographic contrasts applied. This list was taken away and participants were shown a second list of seven names typeset in a single typeface that included distractor names, replacing some of the original names with their look-alike/soundalike counterparts. Participants were asked to identify names they remembered from the first list. In a second test (Figure 10), participants were asked their to give their opinion regarding the ease in differentiating between drug names within a label context.

It was expected that participants would be more likely to accurately identify drug names with the version containing white characters on a black rectangle to differentiate parts of the name, than those that use boldface characters or uppercase characters. Also, it was expected that the version with boldface lowercase characters would be easier to identify than the version with uppercase characters, because of the higher contrast in stroke 
Figure 9. Final designs chosen for testing. Final designs for testing were selected on the basis of legibility and the capacity to create emphasis.

\section{HydrOXYzine HydrALAzine}

\section{Hydroxyzine Hydralazine}

\section{Hydroxyzine Hydralazine}

Figure 10. Variations in a label context
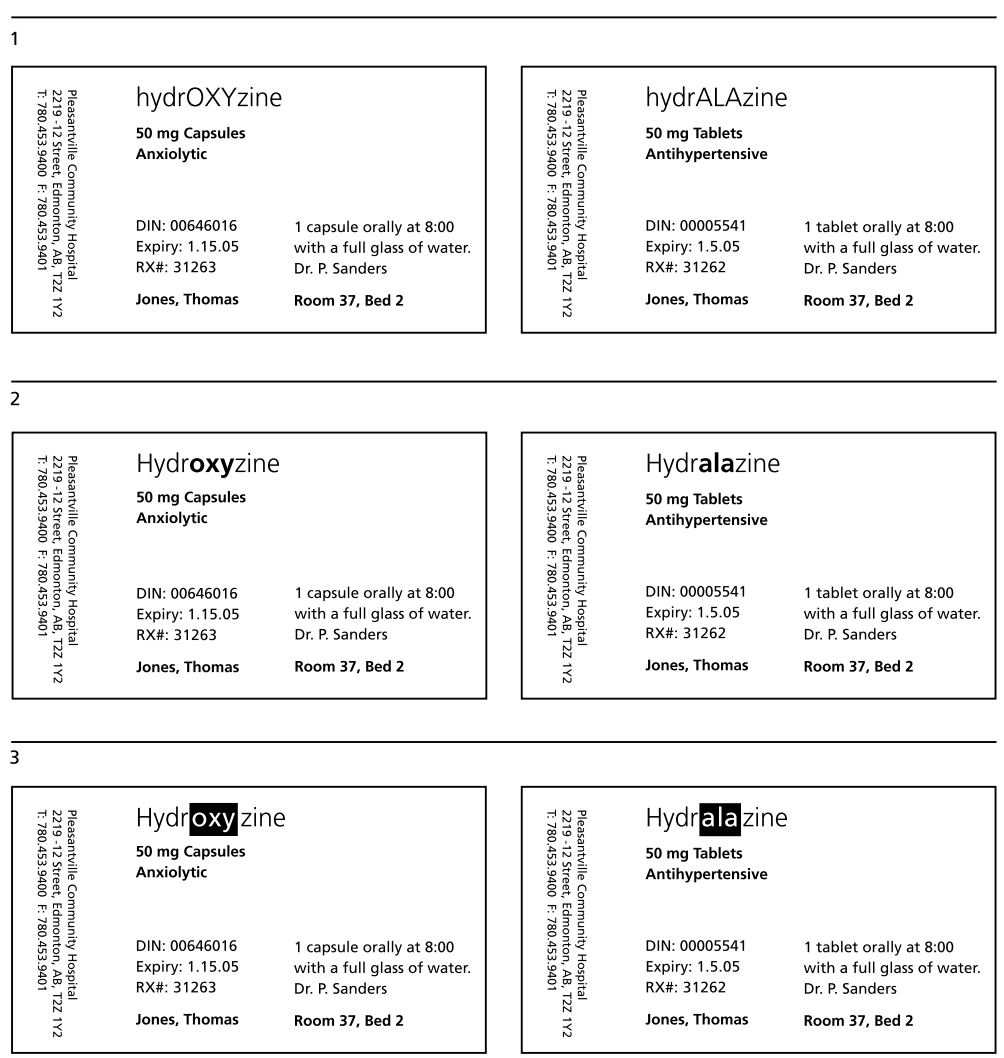

weight and the variation in design that occurs in lowercase characters. The same outcomes were expected in terms of what nurses would perceive to be the easiest to distinguish.

\section{Results}

With the word-recognition tests, contrary to the expected outcomes, participants recognized more names with the use of uppercase characters than with boldface characters. However, as expected, white characters on the black rectangle seem to be most helpful in differentiating names (Figure 11). Consistently with the expected outcomes, when participants were asked which versions were better at helping to distinguish names, comments indicated that most of them perceived that differentiating the name with uppercase characters did not make the names distinctive enough. Opinions were split evenly on the versions that used the boldface characters and those that used white characters on a black rectangle (Figure 12).

\section{A Look to the Future}

This study was concerned with the problem of errors in medicine caused by confusion with look-alike/sound-alike drug names and how the application of typographic choices might help to minimize their occurrence. Though limited in sample size, the testing conducted with the end users indicated that the methods developed could be used with success in a larger study. In terms of effectiveness with the use of typographic contrast to help differentiate look-alike/sound-alike drug names, this study indicated that a stronger degree of contrast than that provided by "tallman letters," specifically, white text on a black rectangle, might help to make names more recognizable. Further research with a larger sample size is required to make concrete recommendations. While word-recognition tests are helpful in comparing the difference that typographic contrasts make in making names more or less memorable, further research should include task-based testing that simulates the medication process and the testing of names in their context, on label prototypes.

To promote safety in the healthcare environment, every effort should be made to help minimize risk to patients. A positive step toward this end would be to incorporate visual communication design practices, and specifically typographic principles and legibility studies, in the production of packaging and labelling. 
Figure 11. Results of word-recognition tests

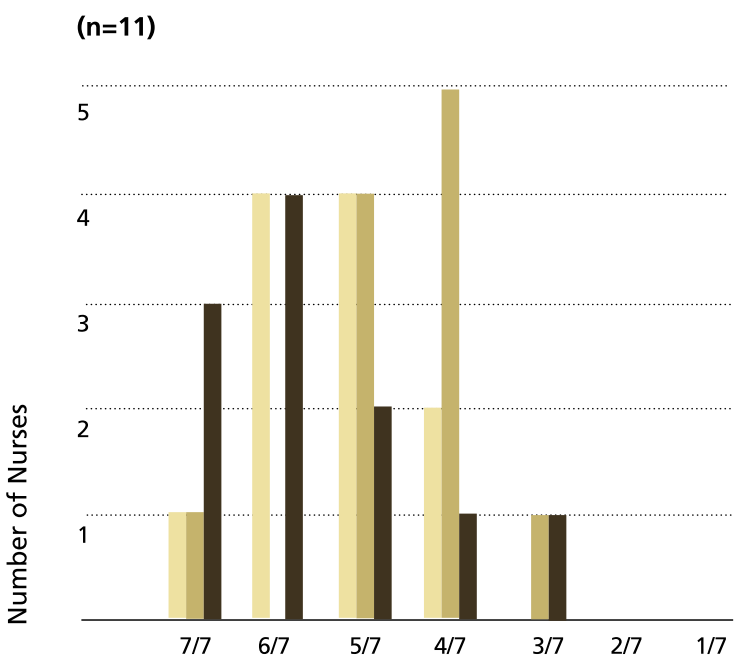

Number of correct responses

$$
\begin{aligned}
& \text { Uppercase characters } \\
& \text { Bold face characters } \\
& \text { White characters on a black } \\
& \text { rectangle }
\end{aligned}
$$

Word recognition tests, comparing the performance of typographic variations to help differentiate look-alike/sound-alike drug names.

Figure 12. Results of test of perception regarding use of typographic variations

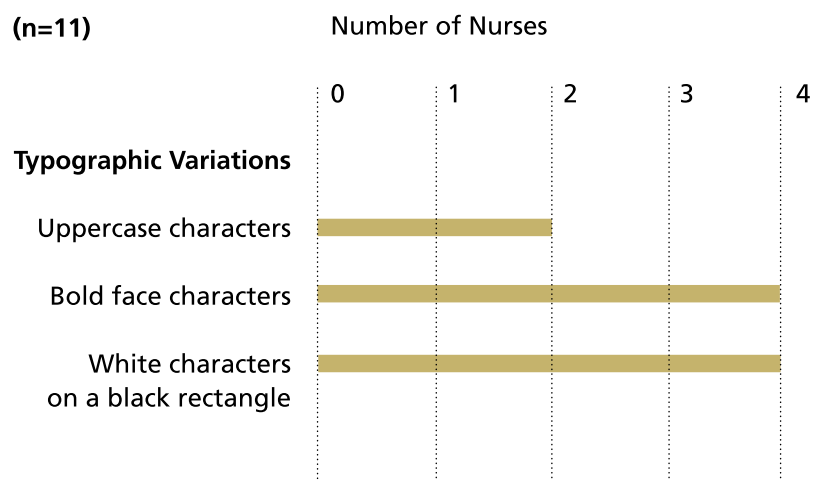

Note: One nurse expressed the opinion that none of the choices helped to differentiate names

Test comparing the perception of nurses regarding the use of typographic variations to help differentiate look-alike/sound-alike drug names.

\author{
About the Author \\ Sandra Gabriele, MDes, RGD, is Assistant Professor, \\ Department of Design, Faculty of Fine Arts, at York University.
}

Please direct correspondence to: Sandra Gabriele, Department of Design, Faculty of Fine Arts, York University, 4700 Keele Street, Toronto, ON M3J 1P3. E-mail: sandrag@yorku.ca.

A special thanks to Valentina Jelincic for her advice during the course of this study.

\section{References}

Baker, G.R., P.G. Norton, V. Flintoft, R. Blais, A. Brown, J. Cox, E. Etchells, W.A. Ghali, P. Hebert, S.R. Majumdar, M. O’Beirne, L. Palacios-Derflingher, R.J. Reid, S. Sheps and R. Tamblyn. 2004. "The Canadian Adverse Events Study: The Incidence of Adverse Events among Hospital Patients in Canada." Canadian Medical Association Journal 170(11): 1678-96.

Biologics and Genetic Therapies Directorate (BGTD), Health Products and Food Branch (HPFB). 2003. Look-Alike Sound-Alike Health Product Names. Ottawa: Health Canada, LA/SA Working Group. Available Health Canada site. Retrieved August 20, 2006. <http:// www.hc-sc.gc.ca/dhp-mps/brgtherap/proj/alike-semblable/lasa-pspcs_ com_response-reponse_e.html>

Broadbent, D.E. 1958. Perception and Communication. New York: Pergamon Press.

Dair, C. 1967. Design with Type. Toronto: University of Toronto Press.

Deutsch, J.A. and D. Deutsch. 1963. "Attention: Some Theoretical Considerations.” Psychological Review 70(1): 80-90.

FDA (United States Food and Drug Administration), Center for Drug Evaluation and Research (CDER). 2002. Medication Errors: Name Differentiation Project. Retrieved August 20, 2006. <http://www.fda. gov/cder/drug/MedErrors/nameDiff.htm>

Filik, R., K. Purdy, A. Gale and D. Gerrett. 2006. "Labeling of Medicines and Patient Safety: Evaluating Methods of Reducing Drug Name Confusion." Human Factors 48(1): 39-47.

Haley, A. [n.d.]. "It's About Legibility." Fonts.com. Retrieved March 7, 2005. <http://www.fonts.com/AboutFonts/Articles/Typography/ Legibility.htm>

Kahneman, D. 1973. Attention and Effort. Englewood Cliffs, NJ: Prentice-Hall.

Kohn, L.T., J.M. Corrigan and M.S. Donaldson (Eds.). 2000. To Err Is Human: Building a Safer Health System. Washington, DC: National Academy Press, Committee on Quality of Health Care in America, Institute of Medicine.

Larson, K. 2004. The Science of Word Recognition: Or How I Learned to Stop Worrying and Love the Bouma. July. Microsoft Typography site. Retrieved August 20, 2006. <http://www.microsoft.com/typography/ ctfonts/WordRecognition.aspx>

Lupton, E. 2003. The Science of Typography. Retrieved August 15, 2004. <http://www.designwritingresearch.org/essays/science.html.>

McClelland, J.L. and D.E. Rumelhart. 1981. "An Interactive Activation Model of Context Effects in Letter Perception: Part 1. An Account of Basic Findings." Psychological Review 88: 375-407. 
McCreight, T. 1996. Design Language. Cape Elizabeth: Brynmorgen Press, Inc.

Norman, D.A. 1990. The Design of Everyday Things. New York: Doubleday.

Pettersson, R. 2001. "Attention: An Information Design Perspective.” Document Design 2(2): 114-30.

Popovic, V. 1999. "Ergonomics, Product Evaluation Methods and Their Importance in Designing Interactive Artifacts." In W.S. Green and P. Jordan, eds., Human Factors in Product Design: Current Practice and Future Trends. London: Taylor and Francis. Pp. 26-35.

Proctor, R.W. and T. Van Zandt. 1994. "Historical Foundations of Human Factors." In R.W. Proctor, Human Factors in Simple and Complex Systems. Boston: Allyn \& Bacon.

Reason, J. 2000a. "Human Error: Models and Management." British Medical Journal 320: 768-70.

Reason, J. 2000b. "Understanding Adverse Events: Human Factors." In C. Vincent, ed., Clinical Risk Management, 2nd ed. London, UK: BMJ Publishing Group. Pp. 31-54.

Rüegg, R. 1989. Basic Typography: Design with Characters. New York, NY: Van Nostrand Reinhold.

Thomas, M.R., C. Hoquist and J. Phillips. 2001. "Med Error Reports to FDA Show a Mixed Bag." Drug Topics 2001 145(19): 23. Available Center for Drug Evaluation and Research, U.S. Food and Drug Administration site. Retrieved August 20, 2006. <http://www.fda.gov/cder/drug/mederrors/reports.htm>

Treisman, A. 1964. "Monitoring and Storage of Irrelevant Messages in Selective Attention." Journal of Verbal Learning and Verbal Behavior 3: 449-59.

U.S. Pharmacopeia (USP). 1998. "Patient Safety.” USP Quality Review 62. Available U.S. Pharmacopeia site. Retrieved August 20, 2006. <http://www.usp.org/patientSafety/newsletters/qualityReview/qr621998-05-01.html>

Van Zandt, T. 1994. Human Factors in Simple and Complex Systems. Boston: Allyn and Bacon.

\section{"We believe our first responsibility is to the doctors, nurses and} patients, to mothers and fathers and all others who use our products and services.

\section{In meeting their needs everything we do must be of high quality."}

- From the Johnson \& Johnson Credo

\section{What are you listening to?}

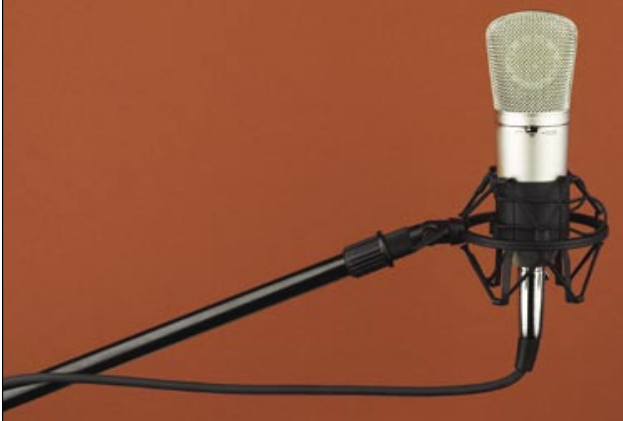

PRODUITS MÉDICAUX

Gohnsonafohnson

MEDICAL PRODUCTS

A DIVISION OF fohmonaffohmon, INC. 